\title{
Locally preferred structure in simple atomic liquids
}

\author{
S. Mossa and G. Tarjus $^{1}$ \\ ${ }^{1}$ Laboratoire de Physique Théorique des Liquides, \\ Université Pierre et Marie Curie, 4 place Jussieu, Paris 75005, France
}

(Dated: March 19, 2018)

\begin{abstract}
We propose a method to determine the locally preferred structure of model liquids. This latter is obtained numerically as the global minimum of the effective energy surface of clusters formed by small numbers of particles embedded in a liquid-like environment. The effective energy is the sum of the intra-cluster interaction potential and of an external field that describes the influence of the embedding bulk liquid at a mean-field level. Doing so we minimize the surface effects present in isolated clusters without introducing the full blown geometrical frustration present in bulk condensed phases. We find that the locally preferred structure of the Lennard-Jones liquid is an icosahedron, and that the liquid-like environment only slightly reduces the relative stability of the icosahedral cluster. The influence of the boundary conditions on the nature of the ground-state configuration of Lennard-Jones clusters is also discussed.
\end{abstract}




\section{INTRODUCTION}

A liquid is said to be supercooled when it is possible to cool it below its melting temperature $T_{m}$ without crystallizing. The supercooled liquid phase is metastable with respect to the underlying crystal, and it is characterized by a dramatic increase of the viscosity and the relaxation times upon lowering the temperature, an increase that eventually leads to glass formation. These dramatic changes in the dynamic properties are not accompanied by strong signatures in the structural quantities, such as the static structure factor. Yet, it has been suggested that both supercooling and glass formation were deeply connected to the structure of the liquid, more precisely to a competition between extension of a local liquid order, different than that of the crystal, and global constraints associated with tiling of the entire space [1, 2, 3]. This competition has been termed geometric (or topological) frustration [1, 2].

Some fifty years ago, Frank put forward the following argument to explain supercooling of liquids 4]. If one considers atomic liquids in which atoms interact via spherically symmetric potentials like the Lennard-Jones potential, the local arrangement of the atoms that is preferred is not the clusters associated with crystalline order (face-centered cubic and hexagonal close-packed lattices), but a polytetrahedral packing, the icosahedron formed by 13 atoms. The energy of such an icosahedral arrangement interacting via the Lennard-Jones potential is indeed $8.4 \%$ lower than the close-packed crystalline clusters. Local icosahedral order should then be prevalent in liquids but, because of the 5 -fold rotational symmetry of the icosahedron, it cannot tile the entire space and form a crystal: this is a manifestation of geometrical frustration. Crystallization then requires a rearrangement of the local structure of the liquid, which leads to a strong first-order freezing transition and allows supercooling of the liquid. Since then, there has been a large body of experimental and simulation work confirming the prevalence of local icosahedral, or more generally polytetrahedral, order in atomic liquids and metallic glasses [2, 5, 6, 7, 8, 9, 10, 11, 12, 13, 14, 15]. The tendency to form icosahedral order has been shown to increase as the temperature is lowered [2, 9, 10, 11, 12, 13, 14, 15].

Frank's argument has, however, one shortcoming [16]: the 13-atom cluster considered is isolated, so that most of the energetics is related to the surface, a situation that of course does not occur in bulk liquids. How can one remedy this problem? An a priori easy way 
would be to study directly the local arrangement of the atoms in a bulk liquid. In such a case, however, it is found that the proportion of icosahedra among all the 13-atom groups is very small, typically a few percent [10]. This is indeed to be expected since geometrical frustration is present, which opposes the growth of icosahedral order and distorts the local polytetrahedral arrangements 2, 17]. How to disentangle then the determination of the locally preferred structure from frustration effects? The method we propose in this work is to consider the influence of the bulk liquid on a given 13-atom cluster at some meanfield level, so that surface effects can be reduced and made more realistic for describing a condensed phase, whereas geometrical frustration is strongly inhibited. The main advantage of this method is that it can be extended to study the locally preferred structure of molecular liquids, for which a priori topological arguments do not easily provide the symmetries of all possible local arrangements of the molecules, nor the nature of geometrical frustration.

More specifically, in this article we consider the ground-state of a cluster of 13 atoms interacting via a Lennard-Jones potential (smaller and larger clusters are also considered); the atoms are placed in a cavity and are subject to an external field that mimics the interaction with the rest of the liquid. The structure of the outside liquid only enters the calculation via the bulk pair distribution function (known from previous simulation studies). By means of an optimization algorithm [18, 19] we find that the ground-state of the cluster, i.e., the global minimum of the (effective) energy surface formed by the intra-cluster interactions and the external field, is of icosahedral symmetry, therefore generalizing Frank's result. For sake of comparison we consider in addition other boundary conditions for the cluster. These conditions describe different types of environments: free boundary conditions (isolated cluster), periodic boundary conditions (periodic tiling of space), and icosahedral-like boundary conditions (hypothetical non-frustrated system).

\section{METHOD AND CHOICE OF THE BOUNDARY CONDITIONS}

We consider a system of atoms interacting via a pair-wise additive spherically symmetric potential $v(r)$, where $r$ is the center-to-center distance. A number $N$ of atoms are placed in a spherical cavity $\mathcal{C}$ of radius $R_{C}$, that we envisage as surrounded by bulk liquid made of the same atoms, and characterized by the temperature $T$ and the density $\rho$. As explained above, we do not want to fully account for the liquid structure because geometrical frustration would 
obscure the nature of the local order. We rather resort to a mean-field type of description in which the liquid outside the cavity is considered as a continuum, characterized by a (known) pair distribution function $g(r ; T, \rho)$ that is not affected by the fact that a cavity has been carved out. The potential energy acting on a given atom at position $\mathbf{r}$ inside the cavity due to the outside liquid is thus described as

$$
W\left(\mathbf{r} ; R_{C}\right)=\frac{\rho}{2} \int_{\mathbf{r}^{\prime} \notin \mathcal{C}} d^{3} \mathbf{r}^{\prime} g\left(\left|\mathbf{r}-\mathbf{r}^{\prime}\right|\right) v\left(\left|\mathbf{r}-\mathbf{r}^{\prime}\right|\right),
$$

where the integral is over all positions outside the cavity. Taking the center of the cavity as origin and transforming to spherical coordinates, one finds after standard manipulations

$$
W\left(r ; R_{C}\right)=\pi \rho\left\{\int_{R_{C}-r}^{R_{C}+r} d x x^{2} g(x) v(x)\left[1-u\left(x ; r, R_{C}\right)\right]+2 \int_{R_{C}+r}^{\infty} d x x^{2} g(x) v(x)\right\},
$$

where

$$
u\left(x ; r, R_{C}\right)=\left(\frac{R_{C}^{2}-r^{2}-x^{2}}{2 r x}\right) .
$$

Note that $u\left(x=R_{C}-r\right)=-u\left(x=R_{C}+r\right)=1$. The detail of the calculation is given in the Appendix.

In view of the implementation of the optimization algorithm [18, 19], we need an explicit expression for the first derivatives of the external potential, both with respect to $R_{C}$ and $r$; we obtain

$$
\left.\frac{\partial}{\partial R_{C}} W\left(r ; R_{C}\right)\right|_{r}=-\pi \rho\left(\frac{R_{C}}{r}\right) \int_{R_{C}-r}^{R_{C}+r} d x x g(x) v(x)
$$

and

$$
\left.\frac{\partial}{\partial r} W\left(r ; R_{C}\right)\right|_{R_{C}}=\pi \rho \int_{R_{C}-r}^{R_{C}+r} d x x^{2} g(x) v(x)\left(\frac{R_{C}^{2}+r^{2}-x^{2}}{2 x r^{2}}\right) .
$$

The second derivatives, needed for the calculation of the Hessian matrix and the study of the transition states [18, 19], can be obtained in a similar way.

The total potential energy for the $N$ atoms of the embedded cluster is the sum of the atom-atom interaction potentials inside the cavity and of the external potential,

$$
\mathcal{U}\left(\left\{\mathbf{r}_{j}\right\}_{1, \ldots, N} ; R_{C}\right)=\sum_{i>j=1}^{N} v\left(\left|\mathbf{r}_{i}-\mathbf{r}_{j}\right|\right)+\sum_{j=1}^{N} W\left(r_{j} ; R_{C}\right)
$$

where $W\left(r ; R_{C}\right)$ is given by Eqs. (2) and (3). Finding the ground-state configuration for the $N$-atom cluster embedded in a liquid-like environment (at a given $T$ and $\rho$ ) amounts to determining the global minimum of $\mathcal{U}$ with respect to variations of the positions of the $N$ atoms. (Note that $T$ and $\rho$ only enter trough the external potential $W\left(r ; R_{C}\right)$, both 
explicitly (see Eq. (2) ) and through the state-dependence of the pair distribution function $g(r)$.) It is worth stressing that, contrary to the case of an isolated cluster for which the radius of the cavity $R_{C}$ is merely fixed to avoid evaporation of the atoms, $R_{C}$ becomes a relevant variable in a liquid-like environment: to preserve a realistic description of the liquid, $R_{C}$ should adjust to global contractions or expansions of the $N$-atom cluster, which should then be taken into account in the minimization procedure. This point will be further discussed below.

To obtain the lowest energy minimum of $\mathcal{U}$, we have used a slightly modified version of the basin-hopping algorithm introduced by Wales and co-workers [18, 19]. The algorithm consists of a constant-temperature Monte-Carlo simulation performed with an acceptance criterion based not upon the energy of the proposed new configuration, but upon the energy of the closest minimum of the potential energy surface, obtained by a local minimization starting from that configuration. This algorithm turns out to be a very efficient method for exploring directly the minima of the potential energy surface, and it allows one to locate the ground-state with relatively little effort.

Finally we have also considered other boundary conditions for the $N$-atom cluster:

i) Free boundary conditions (isolated cluster).- This is the standard case studied in the literature. It simply corresponds to the above situation in which the external potential is set to zero:

$$
W\left(r ; R_{C}\right)=0 .
$$

ii) Periodic boundary conditions (periodic replication of the local cluster).- Each atom of the cluster now interacts also with the images of the other atoms of the cluster

$$
W(\mathbf{r})=\frac{1}{2} \sum_{j \in \mathcal{C}} v\left(\left|\mathbf{r}-\mathbf{s}_{j}\right|\right),
$$

where $\mathbf{s}_{j}$ is the position of the image of atom $j$ selected through the minimum image criterion, i.e., among all possible images of atom $j$, only the closest is selected. We have used a cubic elementary cell.

iii) Icosahedral-like boundary conditions (hypothetical non-frustrated system).- As mentioned in the Introduction, icosahedral order cannot be extended to the entire space. One can however introduce icosahedral-like boundary conditions by embedding the 13-atom cluster in the center of a large 147-atom cluster with icosahedral $\left(I_{h}\right)$ symmetry in which the central atom and its first layer have been removed. (Recall that atomic clusters are characterized 
by "magic numbers" of atoms for which the global minimum has polytetrahedral symmetry 20, 21]; in particular, icosahedral symmetry is obtained for 13, 55, and 147 atoms.) The external potential is now written as

$$
W(\mathbf{r})=\frac{1}{2} \sum_{k \in S_{2}^{i c o}, S_{3}^{i c o}} v\left(\left|\mathbf{r}-\mathbf{r}_{k}\right|\right)
$$

where $S_{2}^{i c o}$ and $S_{3}^{i c o}$ are the second and third shells of the 147-atom icosahedron. For completeness, we have also considered the 55-atom Mackay icosahedron, as well as the correction due to embedding the 55-atom or 147-atom icosahedron in bulk liquid with the resulting external potential treated at a mean-field level (see above).

\section{GLOBAL MINIMUM OF THE LENNARD-JONES CLUSTERS}

We specialize our investigation to the case of the Lennard-Jones pair potential

$$
v_{L J}(r)=4 \epsilon\left[\left(\frac{\sigma}{r}\right)^{12}-\left(\frac{\sigma}{r}\right)^{6}\right]
$$

where $\epsilon$ and $2^{1 / 6} \sigma$ are the well-depth and the separation at the minimum of the potential, respectively. In what follows we set $\sigma=\epsilon=1$.

In order to evaluate the liquid-like external potential acting on the $N$-atom cluster, Eqs. (2) and (3), and its derivatives, Eqs. (44) and (5), one needs a model for the pair distribution function $g(r)$. We use the 7-parameter parametrization of Verlet's Molecular Dynamics simulation data on the Lennard-Jones liquid [22] proposed by Matteoli and Mansoori [23]:

$$
g(y)=1+y^{-m}[g(d)-1-\lambda]+\left[\frac{(y-1+\lambda)}{y}\right] \exp [-\alpha(y-1)] \cos [\beta(y-1)],
$$

for $m \geq 1, y \geq 1$, and

$$
g(y)=g(d) \exp \left[-\theta(y-1)^{2}\right]
$$

for $y<1$. Here $y=r / d$ is the dimensionless intermolecular distance where $d=2^{1 / 6} \sigma$, and $h, m, \lambda, \alpha, \beta, \theta, g(d)$ are adjustable parameters. The terms $y^{-m}$ and $\exp \left[-\theta(y-1)^{2}\right]$ describe the decay of the first peak, while the term $\exp [-\alpha(y-1)] \cos [\beta(y-1)]$ provides the damped oscillations observed at larger distances [23]. We have taken the values $h=1.065$, $m=13.42, g(d)=2.830, \lambda=0.9310, \alpha=1.579, \beta=6.886, \theta=135.9$ that allow to 
reproduce the pair distribution function for the liquid at $\rho=0.880$ and $T=1.095$ (in usual reduced Lennard-Jones units) [22, 23].

The resulting external potential $W\left(r ; R_{C}\right)$ is shown in Fig. 1 for several values of the cavity radius $R_{C}$. The shape of the $r$-dependence changes with $R_{C}$ so that no rescaling of the curves is possible. For $R_{C}<1.2 \sigma$, the potential has a minimum at $r=0$ because the central atom sits at the minimum - or very close to it - of the pair interactions due to liquid atoms at the boundary of the cavity; this is no longer true for larger cavity radii, and $W$ decreases monotonically with $r$, the most favorable position inside the cavity being at its edge where the attractive interaction due to the nearby liquid particles is the strongest. By construction, when $R_{C} \rightarrow 0, W\left(r=0, R_{C}\right)$ becomes equal to the total potential energy of the Lennard-Jones liquid at the considered state point, $E \simeq-5.7 \epsilon$, whereas when $R_{C} \rightarrow \infty$, $W\left(r=R_{C}, R_{C}\right)$ is equal to half this energy.

As we discussed it above, the radius of the cavity $R_{C}$ must be adjusted to global contractions or expansions of the cluster. A reasonable way to implement this is to take at each minimization step, i. e., for each configuration of the $N$ atoms,

$$
R_{C}=r_{\max }+\mu \sigma
$$

where $r_{\max }$ is the distance of the outermost atom from the center of the cavity, and $\mu$ is a constant chosen to account for the fact that repulsive interactions between atoms make very unlikely the presence of "bulk liquid" atoms when their centers are too close to those of the cavity atoms; we have taken $\mu=0.5$, but we have checked that the results are independent of the actual value, in runs with different values of $\mu$ between 0.1 and 1.0.

A typical minimization run for a 13-atom cluster in the presence of a mean-field liquid-like environment is shown in Fig. 2, where we have plotted the evolution of the energy and its intra-cluster and external-field contributions (bottom), together with the evolution of the cavity radius $R_{C}$ (top). During the optimization run that starts from a random configuration of 13 atoms, the structure of the cluster becomes more and more compact, and its energy decreases. The final optimized configuration is found to have icosahedral symmetry. Both the final cluster radius, $r_{\text {max }} \simeq 1.08 \sigma$, and the final intra-cluster energy, $U_{\text {intra }} \simeq-44.327 \epsilon$ are identical to those found for the ground-state of the isolated 13-atom cluster [20]. The total energy of the icosahedral cluster in the presence of a liquid-like environment is however much lower (by almost a factor two) because of the external field that compensates for the 
deficiency of nearest neighbors of the 12 surface atoms. We have repeated the procedure for different starting random configurations and we have always obtained the icosahedron with $r_{\text {max }} \simeq 1.08 \sigma$ as the global minimum. The same is true for a whole range of liquid density $\rho$ and temperature $T(0.65 \leq \rho \leq 0.88,0.6 \leq T \leq 3.6)$.

In addition to carrying out a global optimization procedure, one may also make a calculation in the spirit of Frank's pioneering work [4]. We have considered two potential candidates for the ground-state configuration of the 13-atom cluster, namely the icosahedron and the cuboctahedral cluster with $O_{h}$ symmetry that is associated with the face-centered-cubic close-packed lattice and we have compared their energies in a liquid-like environment. The energies of the two, $I_{h}$-symmetric and $O_{h}$-symmetric, clusters are shown in Fig. 3 as a function of the cavity radius $R_{C}$ (or, equivalently, as a function of $r_{\text {max }}$, a unique distance being enough to fully determine the whole cluster once the symmetry, $I_{h}$ or $O_{h}$, is chosen.). The intra-cluster contribution to the energy has a minimum for $r_{\max } \simeq 1.08 \sigma$ for the icosahedron, and $r_{\max } \simeq 1.10 \sigma$ for the $O_{h}$ cluster, whereas the external-field contribution monotonously increases with $R_{C}$ in both cases. One then finds that the icosahedral cluster has a minimum total energy for $r_{\max } \simeq 1.08 \sigma$ and that this energy is $4.8 \%$ lower than the lowest energy found for the $O_{h}$ cluster when $r_{\max } \simeq 1.10 \sigma$. When compared to Frank's result $(8.4 \%)$, the relative energy difference between the two types of clusters is thus not drastically modified: a mean-field liquid-like environment only slightly reduces the relative stability of the icosahedral order. Finally, as a mere check of our global optimization procedure, we have verified that the icosahedral ground-state found here is identical to that discussed above.

The influence of the boundary conditions on the ground-state of a 13-atom cluster can be investigated by using again the optimization algorithm (always starting with a random initial configuration) with the appropriate conditions, free, periodic, and icosahedral-like, described in the previous Section. As already well-known, the ground-state of the isolated cluster is an icosahedron and, as anticipated, that of the cluster in the presence of icosahedrallike boundary conditions is also an icosahedron. The ground-state energies are shown in Table I. and can be compared to that of the icosahedral cluster in a mean-field liquid-like environment. Not surprisingly, this latter is much lower than that of the isolated cluster (see above), but it is higher than that of icosahedra embedded in larger icosahedral structures.

We note on passing that, in the case of an icosahedral-like environment, the change in the structure of the 13-atom cluster from random to icosahedral during the optimization 
run starts from the outside and propagates inward. This is reminiscent of what has been observed in the simulation of gold nanoclusters [24]. There, it has been found that, just after freezing, ordered nanosurfaces with five-fold rotational symmetry are formed, while interior atoms remain in a disordered state. On lowering the temperature, the crystallization of the interior atoms proceeds from the surface toward the core region, eventually producing

an icosahedral structure [24]. This is at variance with the classical picture of homogeneous nucleation and rather represents a surface-induced (heterogeneous) crystallization.

Periodic boundary conditions lead to a quite different picture. Since icosahedra cannot tile space by periodic replication, such conditions should favor the symmetries that allow a complete filling of space with true long-range order. It is indeed what we have found: the global minimum is then a cuboctahedral cluster with $O_{h}$ symmetry, that leads to a face-centered-cubic close-packed lattice when periodically replicated (see Fig. 4); the corresponding ground-state energy is given in Table I and it is found lower than that of an isolated icosahedron - because of the lack of neighbors already mentioned for this latter case - but higher than icosahedra in either liquid-like or icosahedral-like environment.

Finally, we have considered the effect of varying the number of atoms present in the cluster. The main motivation for this study is to check that without a-priori knowledge of the preferred local structure in the presence of a mean-field liquid-like environment, hence of the number of atoms involved in this structure, the global minimization method will help select the proper preferred configuration. This will be important when considering molecular liquids. We have thus studied the global minima of $N$-atom clusters with $N$ ranging from 2 to 23. As shown in Fig. 5, the energy per-atom, the only relevant quantity for comparing local structures in a liquid-like environment, is lowest for the $N=13$ icosahedral groundstate. For small $N$, the mean-field description of the liquid environment is probably too crude to give sensible results, but it is nonetheless significant that for a large range of $N$, the icosahedral cluster is properly selected as the locally preferred structure of the LennardJones liquid.

\section{CONCLUSION}

Local icosahedral order has been found both in bulk condensed phases and in clusters formed by spherical particles. The connection between bulk and cluster studies is however 
obscured by two facts: first, geometrical frustration strongly hinders the spatial extension of local icosahedral order and distorts the local icosahedra in bulk conditions; second, the energetics of isolated clusters is partly determined by surface effects that are of course absent in the bulk. In this work we have tried to bypass those problems. We have proposed to determine the locally preferred structure of a liquid by finding the ground-state configuration of $N$-particle clusters embedded in a liquid-like continuum, characterized by the proper density and pair distribution function of the bulk liquid at the chosen thermodynamic state point. This mean-field-like procedure minimizes the surface effects without introducing full blown geometrical frustration.

In terms of potential energy surface, we have therefore introduced an effective energy surface that contains the usual intra-cluster potential energy contribution plus an external field that accounts for the interaction with the outside liquid at a mean-field level. By a global optimization algorithm we have then located the lowest-energy minimum of the effective energy surface. For Lennard-Jones pair interactions, we have found that the locally preferred structure is indeed an icosahedron, the effect of the liquid-like environment being to only slightly reduce the relative stability of the icosahedral structure when compared to Frank's calculation for an isolated cluster [4]. We have also shown the importance of the boundary conditions used for the cluster: whereas icosahedral-like boundary conditions stabilize even more the local icosahedral cluster, periodic boundary conditions make the cuboctahedral cluster more stable, a consequence of geometrical frustration that prevents tiling of space by icosahedra, and therefore favors long-range order associated with facecentered cubic or hexagonal close-packed lattices.

The present findings for the Lennard-Jones liquid suggest that the proposed method could be efficient as well for determining the locally preferred structure of molecular liquids, in cases where both translational and rotational degrees of freedom are involved, and a-priori knowledge about the putative local order is scarce.

\section{Acknowledgments}

We thank D. J. Wales and F. Sciortino for fruitful comments. 


\section{APPENDIX: MEAN-FIELD LIQUID EXTERNAL POTENTIAL}

Here we give some details about the calculation of the mean-field liquid external potential $W\left(r ; R_{C}\right)$ discussed in Sect. I.

From Eq. (11) the external potential felt by an atom at $\mathbf{r}$, where the origin of the coordinates is chosen as the center of the cavity, can be written as

$$
W\left(r, R_{C}\right)=\frac{\rho}{2} \int_{|\mathbf{x}+\mathbf{r}|>R_{C}} d^{3} x g(x) v(x)
$$

where we have changed the integration variable from $\mathbf{r}^{\prime}$ to $\mathbf{x}=\mathbf{r}-\mathbf{r}^{\prime}$. We now rotate the reference system such that $\mathbf{r} / r=\hat{\mathbf{z}}$ and we translate it so that the new origin is at $P$, the position of the center of the atom under consideration. The new geometry of the problem is shown in Fig. 6. One can easily convince oneself that

$$
W\left(r ; R_{C}\right)=\frac{\rho}{2} \int_{0}^{\pi} d \phi \sin \phi \int_{0}^{2 \pi} d \theta \int_{s(\phi)}^{\infty} d x x^{2} g(x) v(x) .
$$

Given a point inside the cavity, $s$ is its distance from the surface in the direction $\mathbf{x}$. It is simple to check that $s$ is a function of $\phi$ only and is solution of the quadratic equation

$$
s^{2}+(2 r \cos \phi) s+\left(r^{2}-R_{C}\right)^{2}=0
$$

the correct solution is

$$
s(\phi)=-r \cos \phi+\sqrt{r^{2} \cos ^{2} \phi+\left(R_{C}^{2}-r^{2}\right)} .
$$

Introducing the variable $u=\cos \phi$ we obtain

$$
W\left(r ; R_{C}\right)=\pi \rho \int_{-1}^{1} d u \int_{s(u)}^{\infty} d x x^{2} g(x) v(x)
$$

with

$$
s(u)=-r u+\sqrt{r^{2}\left(u^{2}-1\right)+R_{C}^{2}} .
$$

$s(u)$ is such that $s(-1)=R_{C}+r$, and $s(1)=R_{C}-r$. We now change variable from $u$ to $s$, and integrate by parts so that

$$
\begin{aligned}
W\left(r ; R_{C}\right) & =\pi \rho \int_{R_{C}+r}^{R_{C}-r} d s \frac{d u}{d s} \int_{\rho}^{\infty} d x x^{2} g(x) v(x) \\
& =\pi \rho\left\{\left[u(s) \int_{\rho}^{\infty} d x x^{2} g(x) v(x)\right]_{R_{C}+r}^{R_{C}-r}-\int_{R_{C}-r}^{R_{C}+r} d s s^{2} u(s) g(s) v(s)\right\},
\end{aligned}
$$


where

$$
u(s)=\frac{R_{C}^{2}-r^{2}-s^{2}}{2 r s}
$$

and $u\left(s=R_{C}-r\right)=-u\left(s=R_{C}+r\right)=1$. Substituting Eq. (A.8) in Eq. (A.7) and rearranging we obtain Eqs. (2) and (3).

[1] J. F. Sadoc and R. Mosseri, "Geometric Frustration", (Cambridge University Press, Cambridge, 1999).

[2] D. R. Nelson, Phys. Rev. B 28, 5515 (1983); D. R. Nelson and F. Spaepen, Solid State Physics 42, 1 (1989); D. R. Nelson, "Defects and Geometry in Condensed Matter Physics", (Cambridge University Press, Cambridge, 2001).

[3] D. Kivelson, S. A. Kivelson, X. Zhao, Z. Nussinov, and G. Tarjus, Physica A 219, 27 (1995).

[4] F. C. Frank, Proc. Roy. Soc. A 215, 43 (1952).

[5] A. Filipponi, A. Di Cicco, and S. De Panfilis, Phys. Rev. Lett. 83, 560 (1999).

[6] H. Reichert, O. Klein, H. Dosch, M. Denk, V. Honkimäki, T. Lippmann, and G. Reiter, Nature (London) 408, 839 (2000).

[7] T. Shenk, D. Holland-Moritz, V. Simonet, R. Bellissent, and D. M. Herlach, Phys. Rev. Lett. 89, 075507 (2002).

[8] P. J. Steinhardt, D. R. Nelson, and M. Ronchetti, Phys. Rev. B 28, 784 (1983).

[9] H. Jónsson and H. C. Andersen, Phys. Rev. Lett. 60, 2295 (1988).

[10] T. Kondo, K. Tsumuraya, and M. S. Watanabe, J. Chem. Phys. 93, 5182 (1990); T. Kondo and K. Tsumuraya, ibid. 94, 8220 (1991).

[11] F. Yonezawa, Solid State Physics 45, 179 (1991).

[12] S. L. Shumway, A. S. Clarke, and H. Jónsson, J. Chem. Phys. 102, 1796 (1995).

[13] P. Jund, D. Caprion, and R. Jullien, Europhys. Lett. 37, 547 (1997).

[14] M. Dzugutov, S. I. Simdyankin, and F. H. M. Zetterling, Phys. Rev. Lett. 89, 195701 (2002).

[15] J. P. K. Doye, D. J. Wales, F. H. M. Zetterling, and M. Dzugutov, J. Chem. Phys. 118, 2792 (2003).

[16] J. P. K. Doye, D. J. Wales, and S. I. Simdyankin, J. Chem. Soc. Faraday Discuss. 118, 159 (2001). 
[17] S. Sachdev, in "Bond-Orientational Order in Condensed Matter Systems", K. J. Strandburg Ed. (Springer-Verlag, New York, 1992), p. 255.

[18] D. J. Wales and J. P. K. Doye, J. Phys. Chem. A 101, 5111 (1997).

[19] J. P. K. Doye and D. J. Wales, Phys. Rev. Lett. 80, 1357 (1998).

[20] J. A. Northby, J. Chem. Phys. 87, 6166 (1987).

[21] I. A. Harris, R. S. Kidwell, and J. A. Northby, Phys. Rev. Lett. 53, 2390 (1984).

[22] L. Verlet, Phys. Rev. 165, 201 (1968).

[23] E. Matteoli and G. A. Mansoori, J. Chem. Phys. 103, 4672 (1995).

[24] H. -S. Nam, N. M. Hwang, B. D. Yu, and J. -K. Yoon, Phys. Rev. Lett. 89, 275502 (2002). 


\begin{tabular}{|l||c|c|}
\hline Boundary Conditions & Energy $[\epsilon]$ & Point Group \\
\hline \hline Free & -44.327 & $I_{h}$ \\
Periodic & -52.745 & $O_{h}$ \\
Liquid-like & -88.490 & $I_{h}$ \\
Icos. (1 lay.) & -92.980 & $I_{h}$ \\
Icos. (2 lay.s) & -100.142 & $I_{h}$ \\
Icos. (1 lay.) \& Liquid & -101.395 & $I_{h}$ \\
Icos. (2 lay.s) \& Liquid & -102.208 & $I_{h}$ \\
\hline
\end{tabular}

TABLE I: Ground-state energy and symmetry of the 13-atom cluster with various boundary conditions. The icosahedral-like conditions correspond to the 55-atom (1 layer) and 147-atom (2 layers) icosahedral clusters, and the same structures embedded in a mean-field liquid-like environment.

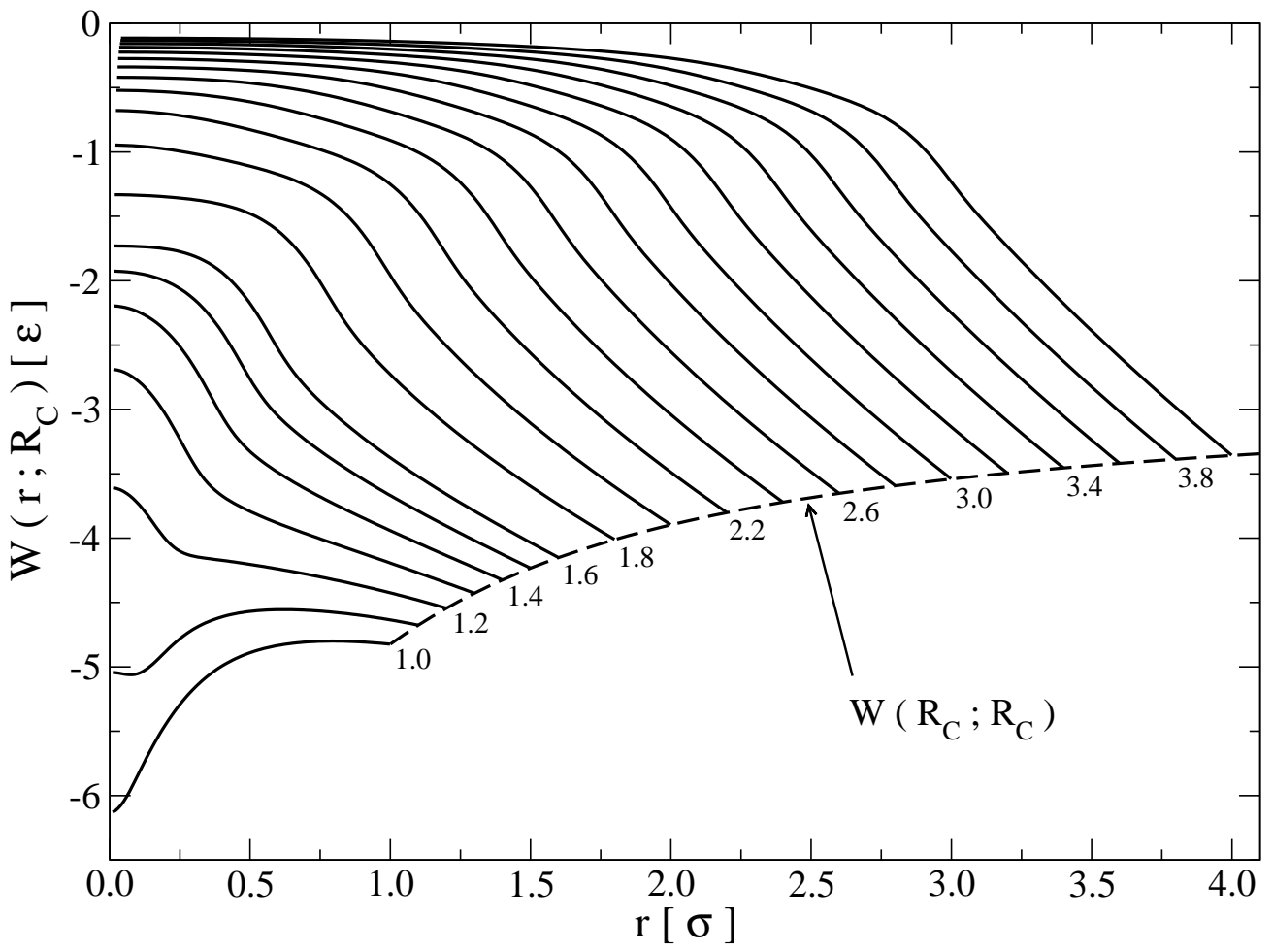

FIG. 1: Mean-field external potential as a function of the distance $r$ from the center of the cavity ; different curves are for different values of the radius of the cavity $R_{C}$. The dotted line is the value of the potential at the surface of the cavity. 


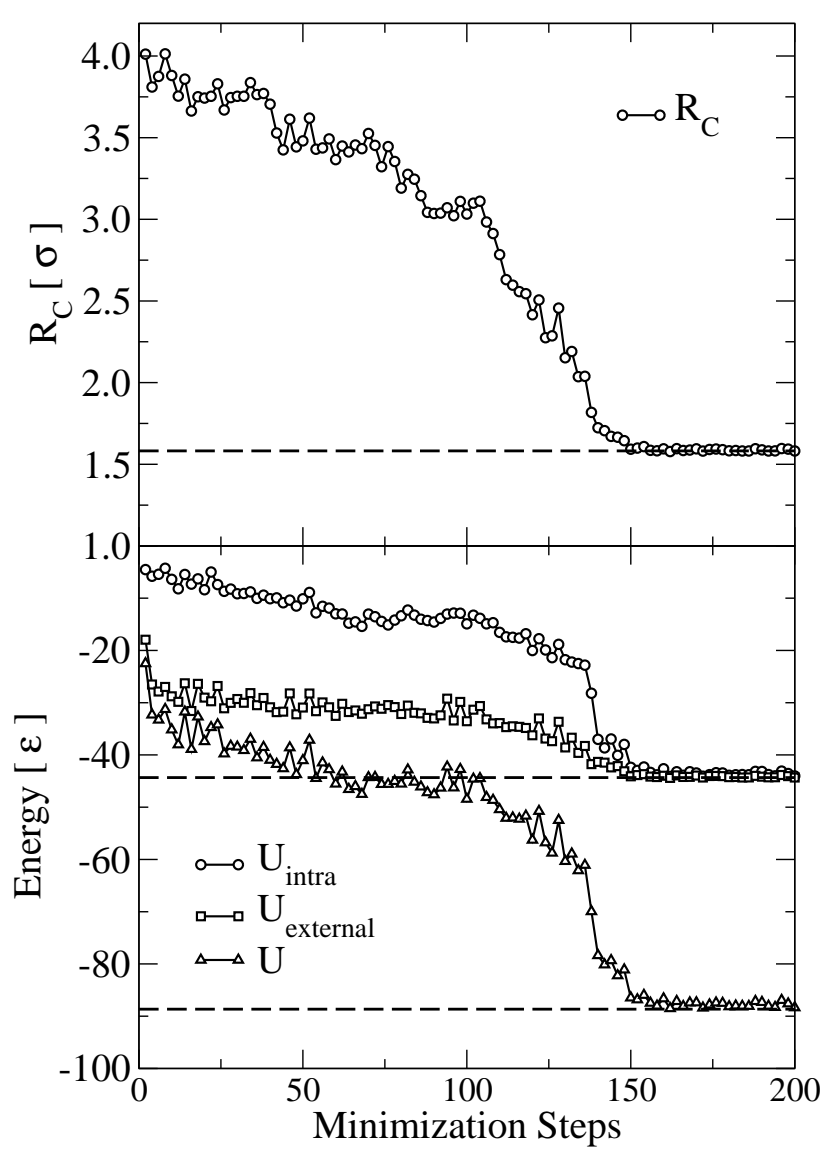

FIG. 2: Evolution of the cavity radius (Top) and of the cluster energy, together with its intra-cluster and external-field contributions (Bottom), during a minimization run starting from a random 13atom Lennard-Jones cluster with mean-field liquid-like environment. 


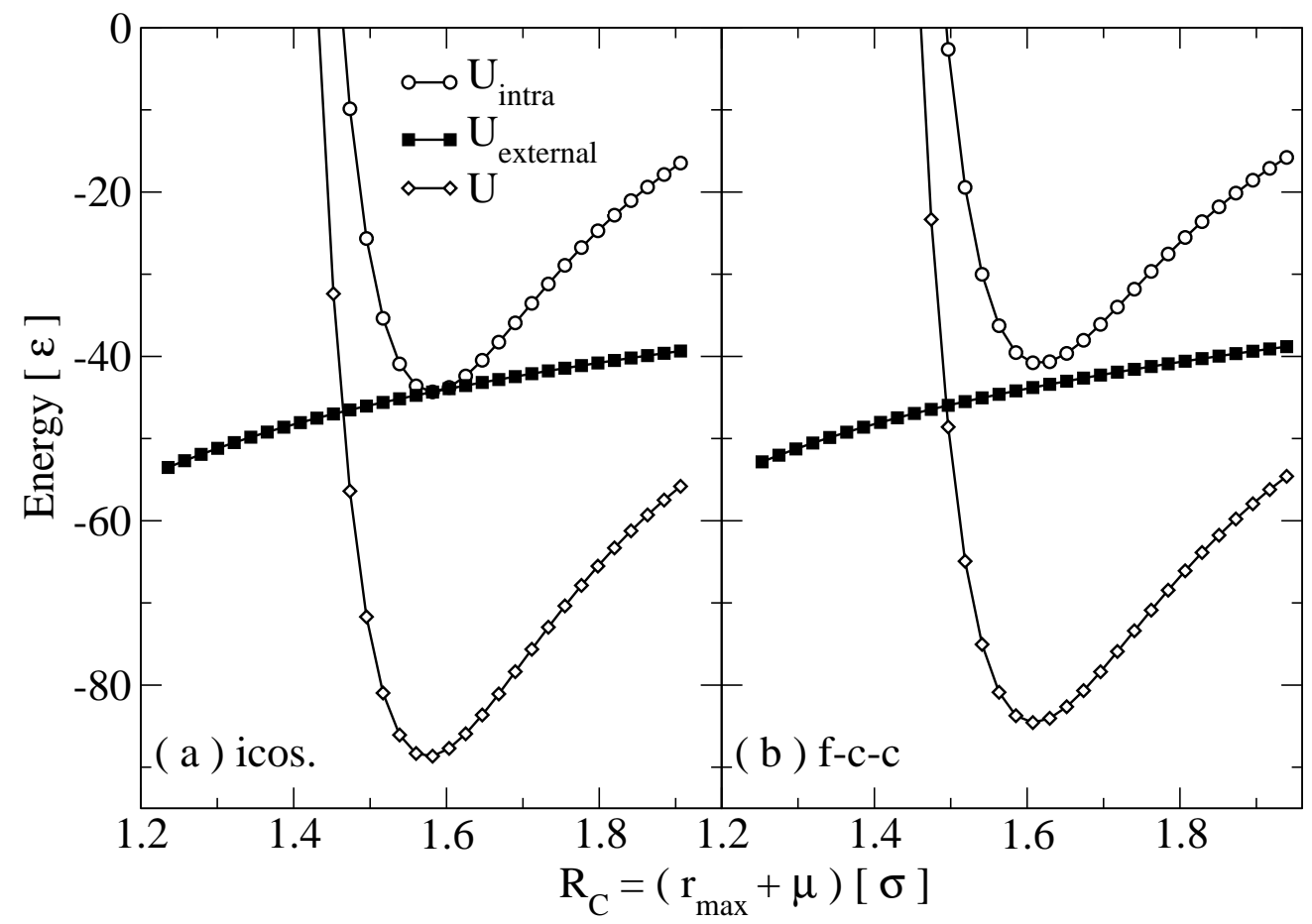

FIG. 3: Energy of a 13-atom cluster with mean-field liquid-like environment as a function of $R_{C}=r_{\max }+\mu \sigma$ (diamonds): (a) icosahedral $\left(I_{h}\right)$ cluster, (b) f-c-c $\left(O_{h}\right)$ cluster. We show separately the contributions of the intra-cluster interaction energy (circles) and that of the external field (squares).

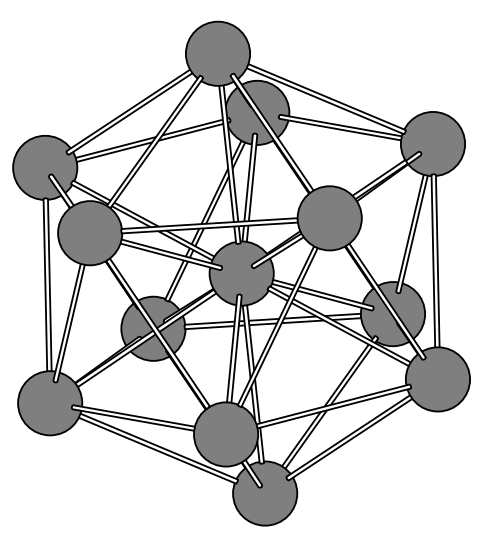

( a ) $I_{h}$ symmetry

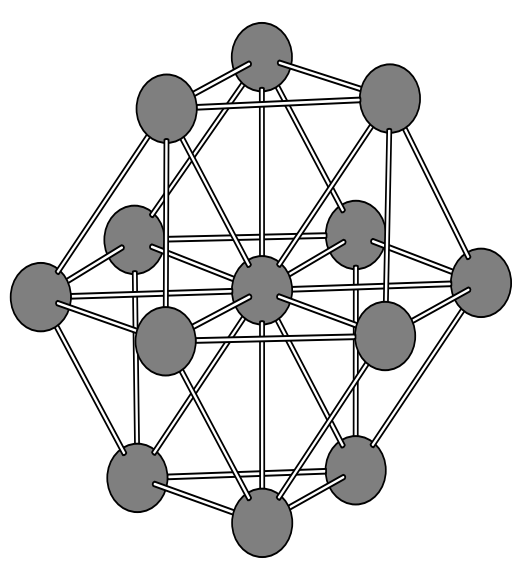

( b ) $\mathrm{O}_{\mathrm{h}}$ symmetry

FIG. 4: Structure of the icosahedral (a) and cuboctahedral (b) 13-atom clusters. 


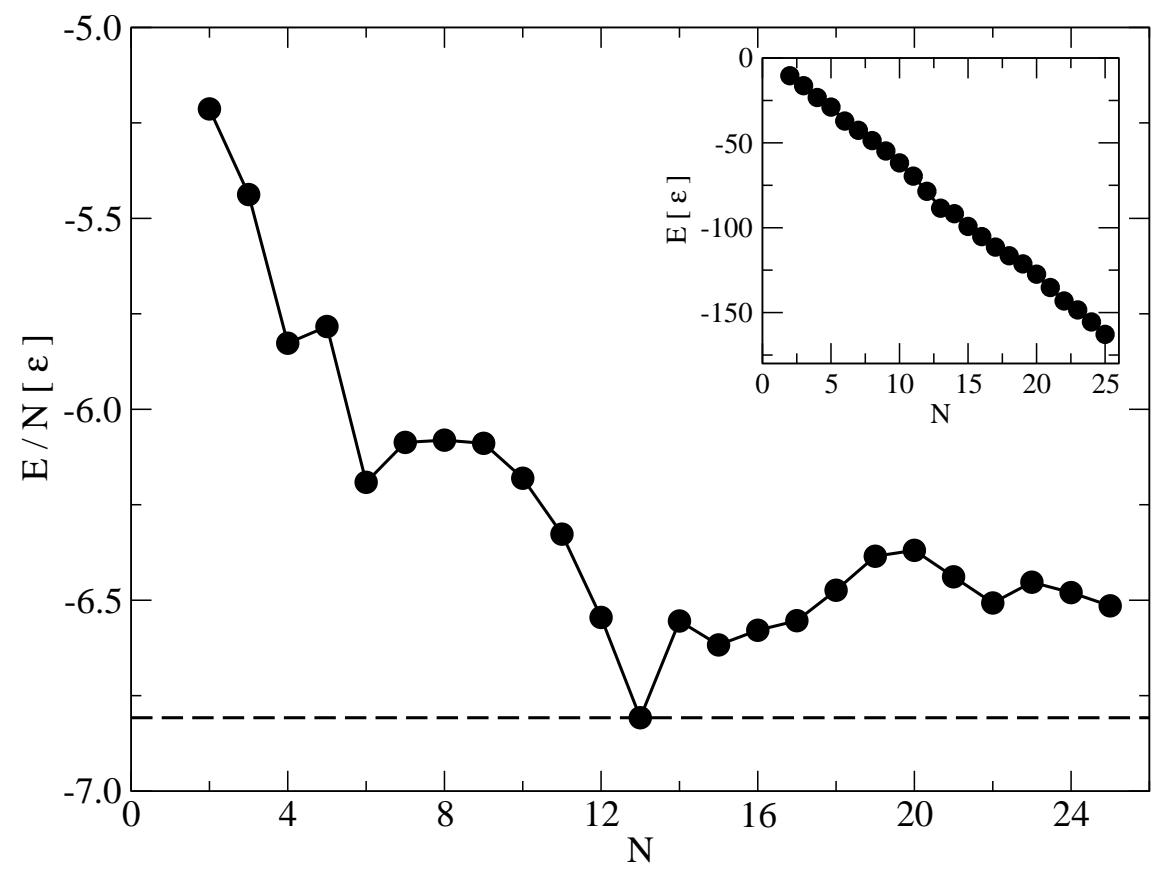

FIG. 5: Energy per atom of the ground-state of $N$-atom clusters with mean-field liquid-like environment. Inset: total energy as a function of $N$.

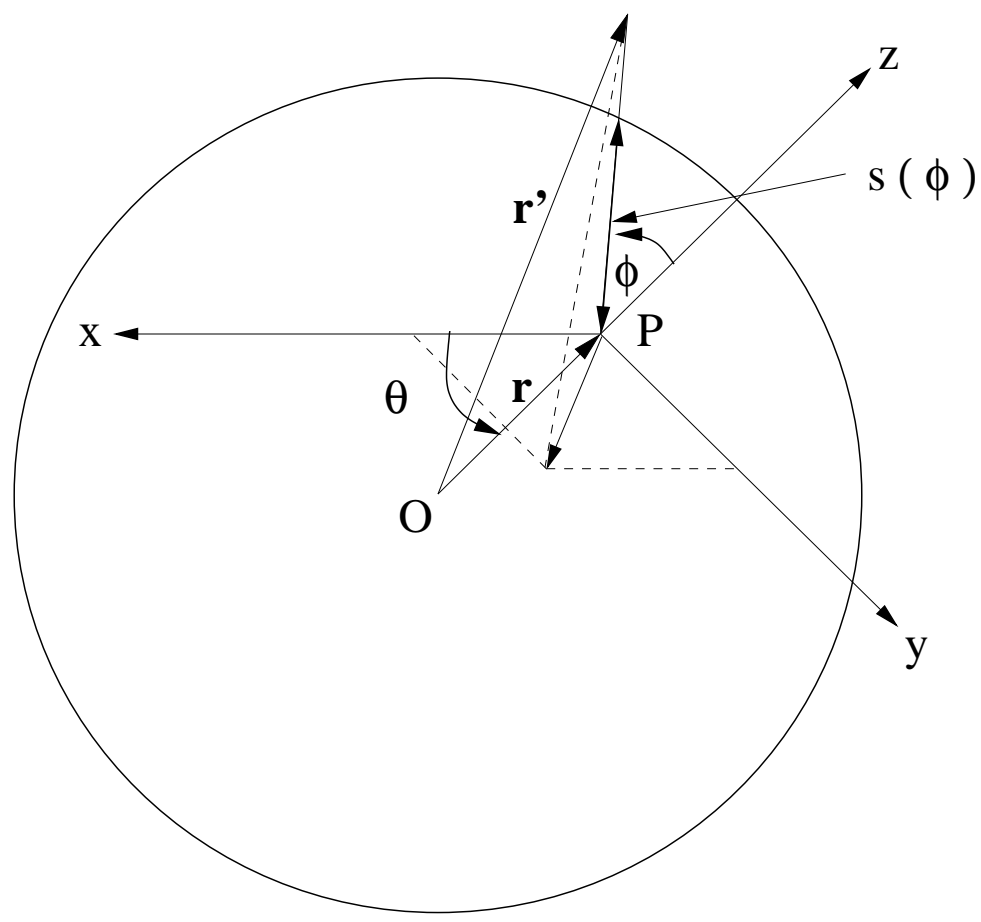

FIG. 6: Geometry used for the calculation of the external potential acting on an atom located at point $P$ in a cavity of radius $R_{C}$. 